\title{
Study on the CAN Search Model based on BDI Agent
}

\author{
Ablimit Arxiden \\ ${ }^{1}$ Faculty of Computer and Information Science, Southwest University, Chongqing 400715, China \\ ${ }^{2}$ Faculty of Mathematics and Information, Teachers College of Hotan 848000 \\ Email: abtht@163.com
}

\begin{abstract}
The main purpose of establishing a complex agent network (CAN) search model is to specifically model each type of the relationships between different types of Agent structure domain and make it easier to be implemented in the existing programming language environment. Under the guidance of complex Agent network method, CAN search process was analyzed, a dynamic search model description was established based on CAN search process, and then individual Agent modelling and the memory and processing of the thinking attributes such as beliefs, desires and intentions in CAN search process were mainly introduced from the individual level; all sorts of Agent conceptual models and Agent type descriptions for CAN search model were designed by introducing BDI Agent; the states and behaviors of the Agent involving in CAN search process were clearly defined.
\end{abstract}

Keywords- Complex Agent Network (CAN); Network Search; Agent Model; BDI Agent

\section{Introduction}

In multi-agent system, BDI model is popular and mature for Agent model design, typically represented by Rao and Georgeff's BDI model and Wooldridge and Jennings' researches. The explanation and analysis by Bratman [1] on its purpose and reason were the philosophical foundations for studying BDI model; the studies of Cohen and Levesque, Rao [2] and Geogeff,
Konolige and Pollack, and Shoham [3] were outstanding. The main tasks in the above studies are concluded as follows: (1) to comprehensively analyze and study Bratman's reason and intentions; (2) to study and define the analysis made by intelligent system [4] on reason and intentions. By emphatically describing the BDI model in the distributed artificial intelligence system, BDI's formal description was studied, and the studies of Cohen and Levesque, Rao [5] and Geogeff, Konolige and Pollack, and Shoham [6] were outstanding. In these studies, Bratman's deep and comprehensive study on reason and intentions was expressed. In this paper, the logic analysis of CAN search strategy and the CAN search model [7, 8] based on BDI Agent were raised. The related work in this paper was done on the basis of the above studies.

\section{BDI Agent architecture}

BDI model consists of the following three parts:

(1) Beliefs

All sorts of Agent thinking and self-beliefs and inner beliefs are integrated together. The way used by Agent to know and understand the world always lies in belief. The belief is composed of various information for describing its own performance and environmental characteristics. The basis of Agent's thinking association is belief.

(2) Desires

The initial target of Agent is the desire, which is an integration of the states that Agent expects to implement or maintain. In real cases, the states 
expected by Agent can motivate it to make corresponding plans and activities.

\section{(3) Intentions}

In all sorts of promised desires, a desire, the most urgent or the most appropriate to implement, is chosen as intention. However, in the application of BDI Agent model information state, how is the behavior process evolved to be the main state? In this paper, a dynamic BDI Agent model and ASL (Agent Script Language) Language [9] were introduced to describe this model: beliefs, desires and intentions had a causal relationship with its behavior, as shown in Fig.1.

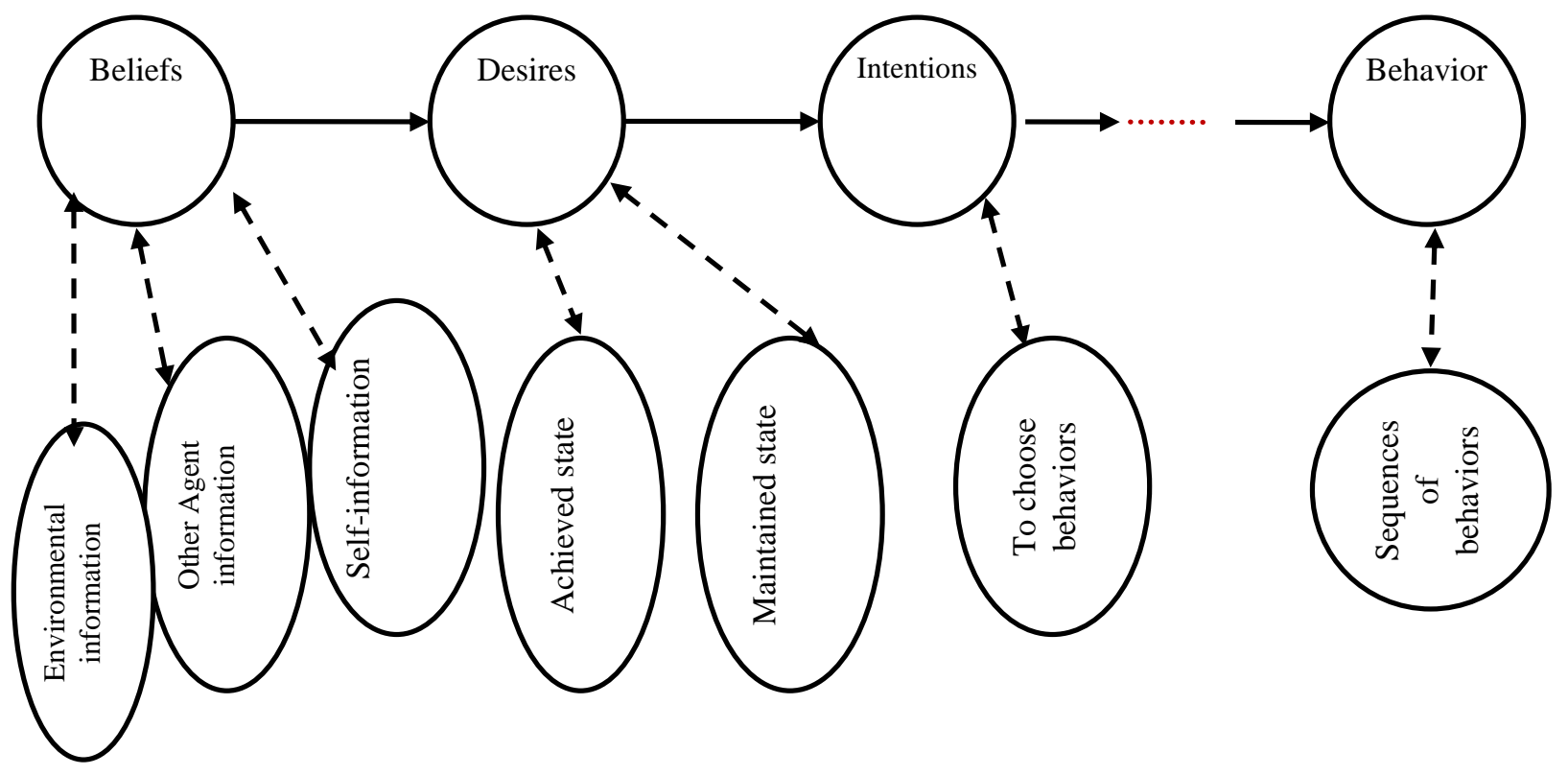

Fig.1 The relation diagram of Beliefs, desires, intentions, and behavior

Cohen et al., after research and analysis according to the philosophy theory of Bratman, thinks that the complete achievement of real intentions needs to abide by the following seven conditions:

(1) Agent has to know how to achieve intentions if it determines tasks according to the intentions

(2) The conflict between Agents is bound not to appear if Agents judge and choose other intentions according to intentions

(3) Agent believes it can achieve self-intention
(4) Agent believes it is voluntary to achieve an intention

(5) Agent tracks the intentions having connections and judges whether the intentions can be achieved, and may choose to try again if the first try fails

(6) Agent thinks intentions can be achieved if special circumstances occur

(7) Some side effect may be caused if Agent does not want to achieve its previously-determined intentions

BDI Agent architecture is as shown in Fig.2. 


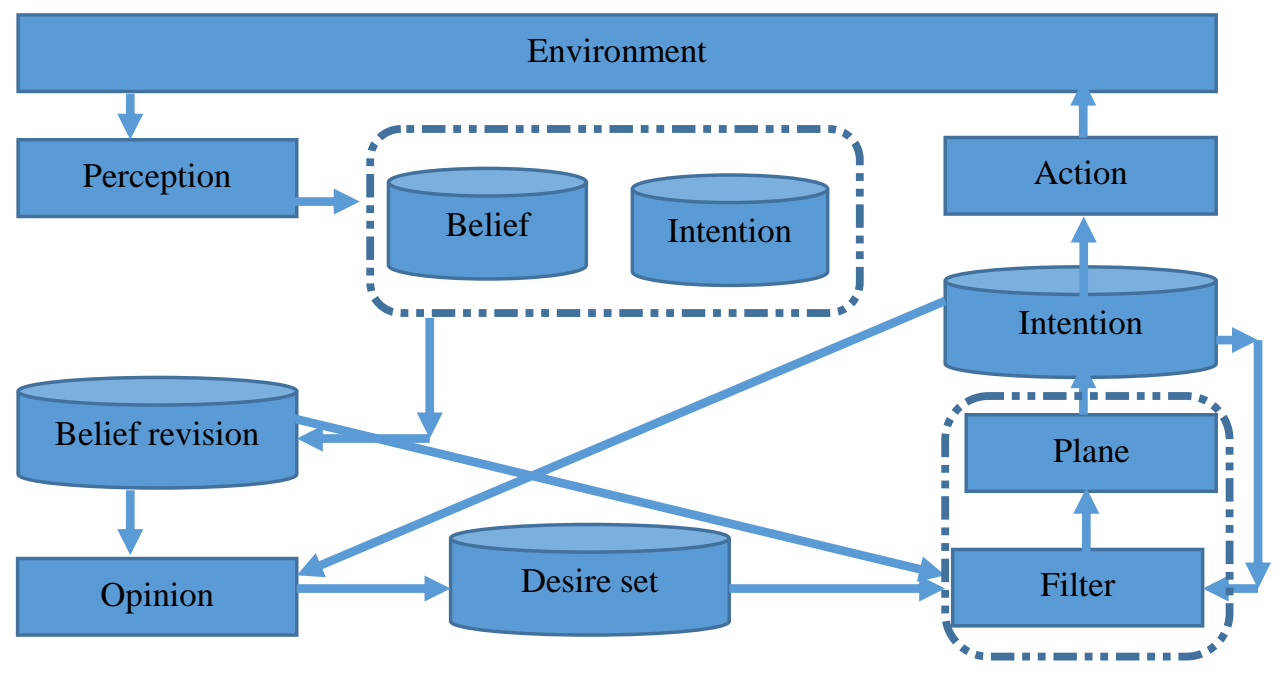

Fig.2 BDI Agent architecture

BDI Agent really simulates the main cause process of mental functional activities, but also leads to complexity, diversity and dynamics. In table 1, the structure was s simplified to target event driven as a cause. The modeling features based on BDI Agent are as follows: seen from the features of BDI Agent, the BDI-based Agent structure more specifically describes the features of intelligent Agent; BDI model also provides the operating features of CAN search model architecture and determines how intentions are produced by desires through communication or event. The main purpose of applying the BDI structure to CAN search model architecture design is as follows:

(1) Intentions can be clearly and explicitly described, making it easy to describe the Agent's knowledge and commitment in CAN

(2) To naturally analyze and design CAN search model

(3) To help to describe and explain the complex behavior of CAN

(4) Possible to help reason the knowledge and behavior of other Agents

\section{The definition of CAN search model}

The attributes of CAN search process state description mainly include searchers, service Agent, search requests, objective resources, query resources, routing tables, pheromone, task lists, node ID, and hop count and so on. 


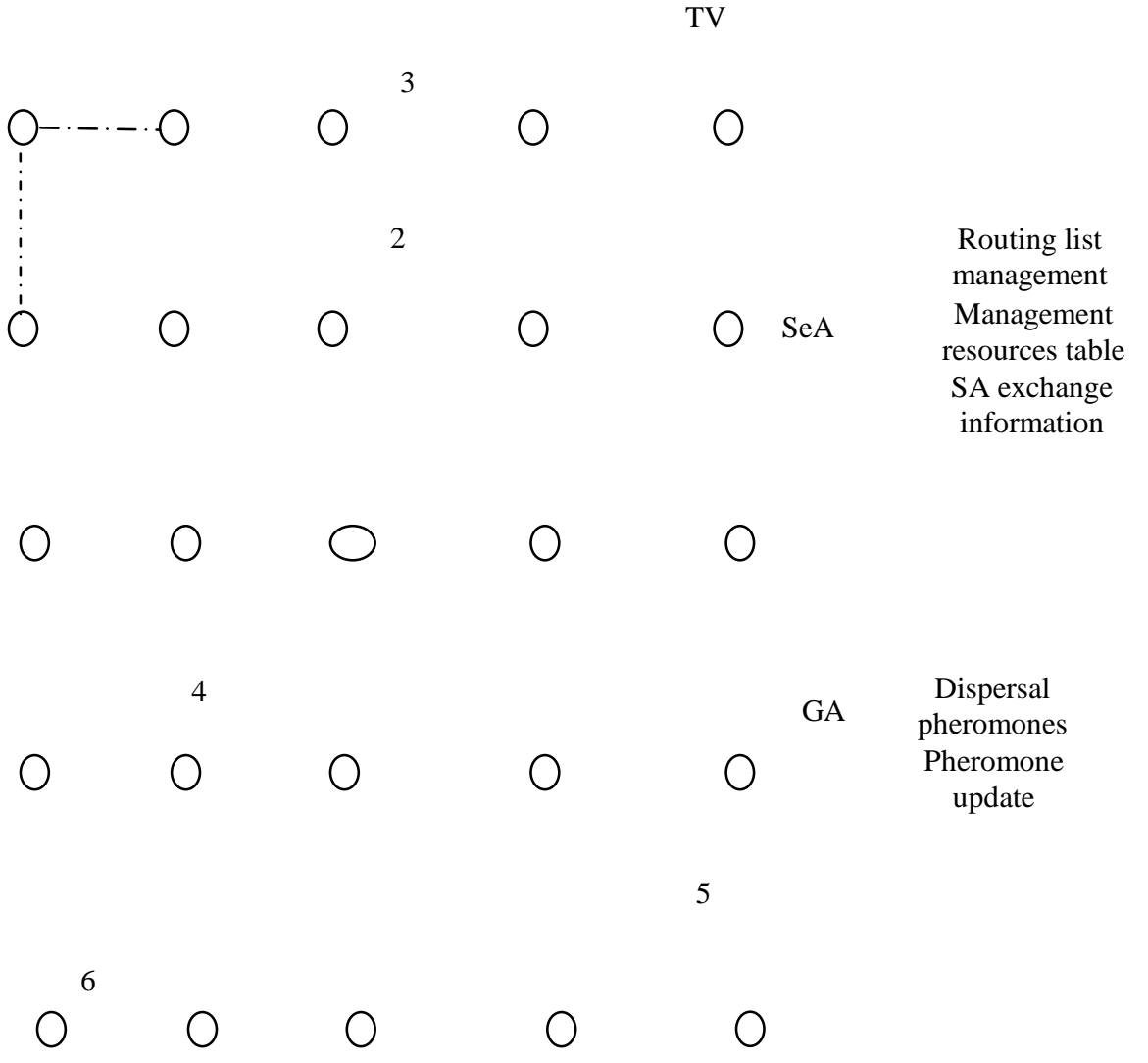

\footnotetext{
Agent node
}

Fig.3 complex Agent network search process description

\subsection{CAN model}

Definition 1 (complex Agent network): It is a model combining multi-Agent system and complex network. Complex Agent network is mathematically defined as follows: a concrete complex Agent network can be abstracted as the diagram composed of Agent node set $V$, frontier set $E$ constituted by the cooperative relations between Agents, and the weight function $W_{\text {Agents }}$ of the close degree of the cooperative relations between Agents.

$$
G_{\text {Agents }}=\left(V_{\text {Agents }}, E_{\text {Agents }}, W_{\text {交互 }}\right)
$$

From mathematical perspective, complex Agent network can be abstracted as a triple.

$$
C A N(t)=\left(V_{\text {Agents }}(t), E_{\text {Agents }}(t), W_{\text {交互 }}(t)\right)
$$


In equation (2), the number of the Agents of node $N$ was $N=|V|$; the number of sides $M$ was $M=|E|$; the weight of side $W$ was $_{W}=|W|$.

A variable $t$ was introduced into the definition of CAN for representing the direct or indirect relations of the node states changes in the set with time. This easily showed the dynamics of complex networks. It is necessary to note that the definition of node was extended to $V_{i}\left(\right.$ Agent $\left._{i}(t), t\right)$; the same sequence $i$ was applied to the sequences of the Agents in multi-Agent system and the sequences of the nodes in complex network. This showed the change of node Agent had connections with time; its self-state change was drive by internal behavior and had close relationship with time. Seen from the above extended definition, node Agent, sides and the two nodes were affected by the Agent. The extended formula could be changed as follows:

$$
\left(V_{i}\left(\operatorname{Agent}_{i}(t), t\right), V_{j}\left(\operatorname{Agent}_{j}(t), t\right)\right)=\left(E_{i}\left(\operatorname{Agent}_{i}(t), t\right), E_{i, j}\left(\operatorname{Agent}_{j}(t), t\right)\right)
$$

In the following, the formulas of all nodes were uniformly abbreviated as $V_{i}^{t}\left(\right.$ Agents $\left._{i}^{t}\right)$ $\underset{E_{i, j}^{t}}{\text { and }}$ Agent $_{i}^{\text {formulas }}$ Agent $\left._{j}^{t}\right)$ of sides were

Definition 2 (private information and public information): What stored in the internal state of Agent is the past information of Agent, which is called as private information. Public information means the information is open to the public. The information available from mutually-related Agents is both local and global.

Definition 3: Complex network has different basic attributes such as degree distribution, average path length, clustering efficient, and Betweenness.

\subsection{Agent model}

Agent model consists of beliefs set and one or more belief states. Predicate, function and the domain of definition could be directly exported from the functional model undertaken by Agent and related areas. In CAN, the Agent belief model participating in the search was formally defined as follows: the belief state $L$ of beliefs set $B$ was the set of real cases in predicates set $P$. After the analysis on the above point of view, it was known that Agent model was composed by the set $S$ of beliefs state $B$ and beliefs states set and the expression was as follows:

$$
\text { Agent }=\left\langle L_{t}, B\right\rangle
$$

Knowledge representation method was determined according to the Agent's existing features to describe Agent to express different beliefs of a concrete Agent model.

Definition 4: $T$ was assumed as the set of identifiers; $P=\left\{p_{1}, p_{2}, \ldots, p_{n}\right\}$ was the set of types domain; $S$ was the set of descriptions on the attributes of beliefs.

- Attribute $H$ was a set of named types domain, and the type of attribute was has $(H)$ that was a ordered pair $\left\langle n, P_{i}\right\rangle$

- A predicate in $\{T, P, S\}$ was expressed with ${ }_{W}\left(S_{1}, \ldots, S_{k}\right)$ that was a tuple $\left\langle w, Y, H_{1}, \ldots, H_{k}\right\rangle$, in which $\quad P \in T, Y \in S$, $\forall_{i}=i, \ldots, k$, has $\left\langle H_{i}\right\rangle \in P$

- A function in $\{T, P, S\}$ was expressed with $f\left(S_{1}, \ldots, S_{k-1}\right) \mid \rightarrow S_{k}$ that was a tuple $\left\langle f, Y, H_{1}, \ldots, H_{k}\right\rangle$, in which $P \in T, Y \in S$, $\forall_{i}=i, \ldots, k$, has $\left\langle H_{i}\right\rangle \in P$.

A set of beliefs was a tuple $\langle T, P, S, W, F\rangle$, in which $W$ was a predicate in $\{T, P, S\} ; F$ was a 
set of functions in $\{T, P, S\}$. Beliefs state was a specific state expressing the upper part of beliefs set; Agent initialization was an initial mental state. Through the above definition, beliefs state and beliefs model could be formally defined as follows

Definition 5: $B=\langle T, P, S, W, F\rangle$ was assumed as a set of beliefs

- A concrete case for predicate $W\left(S_{1} \times, \ldots \times S_{k}\right)$ was that it could be expressed with one tuple:

$W\left(S_{1}, \ldots, S_{k}\right) \in S_{1} \times, \ldots \times S_{k}$

- The beliefs state $L$ of $B$ was the set of predicate cases in $p$, and the belief model was mentioned above

The relations between Agent's beliefs set and events type were discussed below.

Definition 6: $B=\langle T, P, S, W, F\rangle$ was assumed as a set of beliefs, and the beliefs mentioned here were necessarily compatible with the target.
- The set $Q$ of objectives, compatible with ${ }_{B}$, was a set of triple $\langle e, g, O\rangle$, in which $e$ was corresponding to the set of Agent event types and the set of $g \in G$ objective formula; ${ }^{o}$ was the set of the specifiers of the target attributes

- Target state: A target state $U$, compatible with the set of beliefs, was expressed with a set of two-tuples $\langle e, p\rangle$, in which $e$ was corresponding to the set of Agent event types; $p$ was a basic case of a predicate $p \in P$

3.3 Agent description in CAN

Each attribute of Server Agent, especially the attribute closely connected with the search process, constitutes a state domain, including Agent's self-information, the information of other Agents, and environment information. Server Agent state attribute $=\{$ Source list, next hop, hop count, pheromone . Server Agent state attributes are as shown in table 1.

Table 1: The Server Agent state attribute

\begin{tabular}{ccccc}
\hline Vertex ID & Source list & Next Hop & Hop Count & Pheromone \\
\hline$V I$ & $S L$ & $N H$ & $H C$ & $P H$ \\
$v i_{i}$ & $s l_{i}$ & $n h_{i}$ & $h c_{i}$ & $p h_{i}$ \\
\hline
\end{tabular}

SEA behavior $=\{$ Guide, Receive, Manage, update, Spread, Delete $\}$. Server Agent behaviors are as shown in table 2.

Table 2: The Server Agent behavior

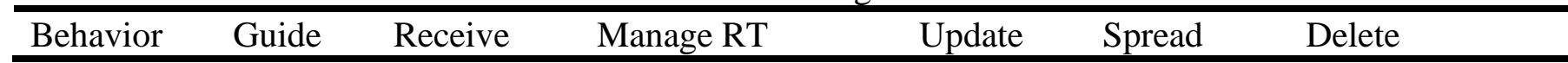

Search Agent roamed on the entire complex Agent network for searching the objective resources in the task list on some network node, specifically including the attribute variables such as Target Source, time to till, hop count, and tabu [10] [11] [12]. TTL was also known as volatilization value, representing the survival time of Agent. (Tabu): the nodes and lines accessed by Agent were recorded in the table for mainly preventing Agent to repeatedly access. Search Agent state $=\{$ Search Agent ID, Source list, Goal source, Time To Till, Next Hop, Hop Count, Tabu, Search Agent $\}$. The state attributes are as shown in table 3.

Table 3: search agent state attribute

\begin{tabular}{lllllll}
\hline Search Agent ID & Source list & Goal source & Time To Till & Next Hop & Hop Count & Tabu \\
\hline SA & SL & $G S$ & $T T L$ & $N H$ & $H C$ & $T A B U$ \\
Sai & sli & $g s_{i}$ & $t l_{i}$ & $n h_{i}$ & $h c_{i}$ & $T a b u_{i}$ \\
\hline
\end{tabular}

Agent behaviors are as shown in table 4. SA behavior $=\{$ Receive, Move, Query, Update, TTL, Res ponce $\}$. 
Table 4: Search agent behavior

\begin{tabular}{|c|c|c|c|c|c|c|}
\hline Receiving task & Move & Choice path & Query & Update & Life Control & Recnonce \\
\hline
\end{tabular}

\section{Description on the search process}

In CAN search, there are a variety of collaborative relations such as full cooperation and selfish between Agents. In Search Agent, different search strategy was applied and this was reflected from cooperation and antagonism. In a different environment, cooperation might provide or convert conditions for search. Then, the role of node Agent in search strategy and the cooperation between Agents were studied. The search is as follows:

Step 1: In a source node on the network, a query request was raised for searching the resource target on the target node

Step 2: A certain number of Search Agent was created (the Search Agent had the basic characteristics of mobile Agent)

Step 3: Search Agent moved according to the preset routing mechanism to query target resources on the neighbor nodes

Step 4: According to the information contents of a task, Search Agent chose the most rational node according to the routing table as the next jump neighbor node

Step 5: Search Agent queried the information resources in each node according to the search strategy and the routing table information and would stop the search when the search resources available from the node were exhausted

Step 6: Search Agent migrated to the next nod according to the migration strategy, and step 4 continues

Step 7: All nodes were completely searched and the search was completed, and the search results were returned to the attributes of the source node

The decision process of Search Agent is as follows:

(1) To determine search problem in CAN system, raise search scheme, and determine the target tasks of Agent
(2) To determine the role of each search state in the successful completion of search strategy

(3) To determine the most effective search state and begin a new search task

(4) To reply search results and determine the second-step search strategy

Agents interaction is an abstract concept and means that Search Agent and node Agent have the same time in CAN and space and search problems may interact and affect each other. Agent cooperation partner is also known as the opponent, which is called as the cooperation model.

\section{Conclusion}

Under the guidance of complex Agent network method, complex Agent network search process was analyzed, a dynamic search model description was established, and then the state factors affecting the search process were analyzed from two levels in complex Agent network search process: the individual Agent modeling and the memory and processing of the thinking attributes such as beliefs, desires, and intentions were mainly introduced; all sorts of Agent conceptual model and Agent type descriptions for the CAN search model were designed by introducing BDI Agent, and the states and behaviors contained by the Agent involving in CAN search process were clearly defined.

\section{References}

[1] Bratman M E. Intentions, Plans, and Practical Reason. Massachusett s: Harvard University Press, 1987.

[2] Cohen P R, Levesque $\mathrm{H} \mathrm{J}$. Intention is Choice with Commitment. Artificial Intelligence, 1990, 42 (2-3): 213 261. 
[3] Rao A S, Georgef f M P. Asymmetry Thesis and Side-effect Problems in linear-time and Branching-time Intention logic: Sridharan N $\mathrm{S}$ ed. Proceedings of the 12th International Joint Conference on Artificial Intelligence. San Mateo, CA: Morgan Kaufmann Publishers, Inc., 1991. 498 504.

[4] Bratman M E. Int ent ions, Plans, and Pract ical Reas on. Massachu sett s: Harvard University Pres s, 1987.

[5] Cohen P R, Levesque H J. Intention is choice with commitment. Artificial Intelligence, 1990, 42(2-3): 213 261.

[6] Rao A S, Georgef f M P. Asymmetry Thesis and Side-effect Problems in Linear-time and Branching-time Intention logic: Sridharan N $\mathrm{S}$ ed. Proceedings of the 12th International Joint Conference on Artificial Intelligence. San Mateo, CA: Morgan Kaufmann Publis hers, Inc., 1991. 498 504.

[7] Ablimit Arxiden. The Modeling of Complex Agent Network based on BDI Agent,
Proceedings of 2015 2ndt International Conference on Electrical and Intelligent, ICEI2015, Volume 9, 94--97, June.28 2015, Boca Raton, USA.

[8] Ablimit Arxiden1,2, Shanxia Wang1 and Huiwen Deng1,*, A Logical Analysis of Complex Network Searching, The Open Cybernetics \& Systemics Journal, 2015,9 (20), 2681- 2687. EI

[9] Shoham Y. Agent-Oriented Programming. Artificial Intelligence, 1993, 60(1): 51-92.

[10] Glover F. Tabu Search: Part I [J]. OR SA J on Com2 p u ting, 1989, 1 (3): 1902206.

[11] L M Gambardella, M Dorigo HAS SOP: A Hybrid Ant System for the Sequential Ordering Problem [R]. IDSIA, Tech Rep: 97-11, 1997.

[12] Dorigo M, Gambardella LM. Ant Colony System: A Cooperative Learning Approach to the Traveling Salesman Problem. IEEE Trans on Evolutionary Computation, 1997, 1(1): 53-66. 